\title{
Acides gras oméga-3 et déclin cognitif : la controverse
}

\author{
Pascale BARBERGER-GATEAU ${ }^{1,2}$ \\ Cécilia SAMIERI ${ }^{1,2}$ \\ Catherine FÉART ${ }^{1,2}$ \\ Stephen CUNNANE ${ }^{3}$ \\ ${ }^{1}$ Univ. Bordeaux, \\ ISPED, \\ Centre INSERM U897-Epidemiologie- \\ Biostatistique, \\ F-33000 Bordeaux, \\ France \\ $<$ Pascale.Barberger-Gateau@isped.u- \\ bordeaux2.fr $>$ \\ 2 INSERM, \\ ISPED, \\ Centre INSERM U897-Epidemiologie- \\ Biostatistique, \\ Université Bordeaux Segalen \\ 146, rue Léo-Saignat, \\ F-33076 Bordeaux cedex, \\ France \\ ${ }^{3}$ Research Center on Aging, \\ Dept of Medicine, \\ Universite de Sherbrooke, \\ Sherbrooke, \\ QC, \\ Canada
}

Article reçu le 30 novembre 2012

Accepté le 5 décembre 2012

Environ une personne âgée sur cinq présente des troubles cognitifs légers après 65 ans (Ritchie et al., 2001), et près d'une sur cinq est atteinte de démence après 75 ans (Ramaroson et al., 2003). Les deux causes les plus fréquentes de démence sont la maladie d'Alzheimer et la démence vasculaire, avec cependant de très nombreuses formes mixtes associant lésions neurodégénératives et vasculaires cérébrales comme le montre

\begin{abstract}
Omega-3 fatty acids and cognitive decline : the controversy
Basic research suggests a protective effect of the long-chain omega-3 polyunsaturated fatty acids - eicosapentaenoic acid (EPA) and docosahexaenoic acid (DHA) - against brain aging. In humans, many epidemiological studies have found an inverse association between fish consumption or high blood levels of EPA and DHA, and cognitive decline or risk of dementia. However, most randomized controlled trials with EPA and/or DHA supplements have failed to show any impact on cognitive decline. This paper analyses several reasons for such inconsistent results, including the time and duration of the supplementation, the cognitive and dietary inclusion criteria, the optimal doses of EPA and DHA, the interaction with genetic polymorphisms, and the need to consider synergistic effects between nutrients as they are provided by healthy diets.
\end{abstract}

Key words: omega-3, EPA, DHA, brain, Alzheimer, cognitive decline, intervention studies, methods

l'imagerie par résonance magnétique (Viswanathan et al., 2009). Par ailleurs, les lésions neurodégénératives de la maladie d'Alzheimer, protéines betaamyloïde et tau hyperphosphorylée, s'accumulent pendant des dizaines d'années dans le cerveau avant que $n$ 'apparaissent les premiers signes de déclin cognitif (Jack JR et al., 2010). La démence du sujet âgé résulte donc d'une interaction entre des facteurs non modifiables comme la prédisposition génétique (notamment l'allèle epsilon 4 du gène de l'apoliporotéine $E, A p o E 4$ ) et des facteurs environnementaux éventuellement modifiables parmi lesquels la nutrition pourrait jouer un rôle majeur. En effet, un excès d'apport énergétique peut contribuer à l'obésité, au syndrome métabolique et au diabète, facteurs de risque vasculaires mais aussi de maladie d'Alzheimer (Profenno et al., 2010). Par ailleurs I'alimentation apporte des nutriments comme les acides gras polyinsaturés oméga-3 à longue chaîne acides eicosapentaénoïque (EPA) et docosahexaénoïque (DHA) - qui pourraient contribuer à protéger le cerveau contre les lésions neurodégénératives et vasculaires, et les phénomènes $d^{\prime}$ inflammation et de stress oxydant qui les accompagnent (Barberger-Gateau et al., 2012). En accord avec les données des études sur des modèles cellulaires ou animaux, de nombreuses études épidémiologiques d'observation ont montré une association inverse entre la consommation de poisson, ou le statut sanguin en EPA et DHA, et le déclin cognitif ou le risque de développer une démence (Barberger Gateau, 2007; Cunnane et al., 2009). Une concentration plasmatique élevée en EPA a même été associée à une moindre atrophie de la matière grise dans le lobe temporal médian, région atteinte de façon spécifique et précoce dans la maladie d'Alzheimer (Samieri et al., 2012).

Malgré ce faisceau d'arguments biologiques et épidémiologiques convaincants, les études d'intervention à type d'essais randomisés avec de l'EPA et/ou du DHA contre placebo se sont avérées très décevantes pour la prévention primaire ou secondaire du déclin cognitif chez le sujet âgé, comme le montre une métaanalyse récente (Mazereeuw et al., 2012). Une revue Cochrane des essais contrôlés randomisés évaluant l'impact d'une supplémentation en oméga-3 a conclu qu'il n'y avait pas d'étude permettant d'évaluer leur impact sur l'incidence de la

Pour citer cet article : Barberger-Gateau P, Samieri C, Féart C, Cunnane S. Acides gras oméga-3 et déclin cognitif : la controverse. OCL 2013 ; 20(2): 88-92. doi : 10.1684/ocl.2012.0495 
démence et que les études d'intervention sur le déclin cognitif ne démontraient pas d'impact significatif (Sydenham et al., 2012). De même, les études d'intervention ayant administré de l'EPA ou du DHA chez des sujets atteints de démence de type Alzheimer n'ont montré aucun bénéfice de cette supplémentation sur le déclin cognitif ou fonctionnel (Cunnane et al., 2009; Quinn et al., 2010). Cependant, en l'état actuel des connaissances, il n'est pas possible de déterminer si ce désaccord entre, d'une part, les études fondamentales et épidémiologiques, et, d'autre part, les études d'intervention, révèle une absence réelle de bénéfice de la supplémentation en oméga-3 sur les fonctions cognitives, ou si ces discordances ne reflètent que les limites méthodologiques des études publiées (Dangour et al., 2012). Les limites des études épidémiologiques sont bien connues, en particulier la possibilité de facteurs de confusion résiduels non contrôlés comme le mode de vie. Cependant, les essais d'intervention se heurtent également à des difficultés méthodologiques qui peuvent en limiter la portée. L'objectif de cette revue est de discuter certaines limites des essais contrôlés randomisés évaluant l'impact des oméga3 sur les fonctions cognitives du sujet âgé à travers une série de questions à se poser avant de concevoir un essai ou en en lisant les résultats publiés.

\section{Quand et qui faudrait-il supplémenter?}

La fenêtre optimale d'exposition à une supplémentation en EPA et DHA n'est pas connue : faut-il inclure des sujets n'ayant pas encore de troubles cognitifs (prévention primaire), avec troubles légers (prévention secondaire) ou déjà déments (thérapeutique) ? Même s'il s'agit d'un continuum, I'histoire naturelle de la maladie d'Alzheimer peut être divisée en trois phases successives selon le modèle proposé par Jack et al. (Jack JR et al., 2010): une longue période préclinique asymptomatique, suivie d'une phase de transition avec des troubles cognitifs légers encore réversibles (mild cognitive impairment ou $\mathrm{MCl}$ ), puis la phase de démence, irréversible, lorsque les troubles cognitifs deviennent suffisamment importants pour retentir sur la vie quotidienne.

La phase préclinique s'étendrait durant plusieurs dizaines d'années pendant lesquelles les lésions neurodégéné- ratives s'accumulent dans le cerveau, s'accompagnant d'anomalies du métabolisme du glucose cérébral, d'inflammation avec une activation de la microglie, et de stress oxydant, sans qu'il existe de plainte de mémoire ni de déficit cognitif objectivable avec la plupart des tests neuropsychologiques usuels. À ce stade, l'impact d'une intervention de prévention primaire du déclin cognitif avec des recommandations alimentaires ou des suppléments d'oméga-3 est particulièrement difficile à évaluer car en l'absence de symptômes, il n'est pas possible d'identifier les sujets les plus à risque de déclin cognitif. En outre, celui-ci ne surviendra que très progressivement, il faudrait donc intervenir pendant des années afin de pouvoir observer une modification de la pente du déclin cognitif et un retard à la survenue d'une démence. L'étude PAQUID a néanmoins pu montrer qu'il existait un déclin à certains tests cognitifs de mémoire sémantique comme le Set Test d'Isaacs dès 12 ans avant que la démence ne soit diagnostiquée (Amieva et al., 2008). La méta-analyse de Mazereeuw (Mazereeuw et al., 2012) a montré que dans le sous-groupe de sujets ayant conservé des fonctions cognitives globales satisfaisantes (minimental state examination (MMSE) > 25), la supplémentation avec de I'EPA ou du DHA avait un effet global sur la mémoire verbale mais pas sur les autres tests cognitifs. Parmi les études incluses dans cette méta-analyse, MIDAS (Memory Improvment with DHA Study) est un essai randomisé contre placebo qui a inclus des sujets relativement jeunes ( 55 ans et plus), ayant une plainte de mémoire subjective avec des fonctions cognitives globales préservées mais des performances moins bonnes que des sujets plus jeunes sur un test de mémoire logique (Yurko-Mauro et al., 2010). Les participants qui ont reçu $900 \mathrm{mg} / \mathrm{j}$ de DHA pur extrait d'algue avaient des performances significativement meilleures que ceux ayant reçu un placebo à plusieurs tests de mémoire mais pas des fonctions exécutives après 24 semaines de supplémentation. Cette étude suggère qu'une intervention précoce pourrait retarder un déclin cognitif extrêmement léger. Au stade de $\mathrm{MCl}$, I'atrophie cérébrale et la perte synaptique s'accompagnent de troubles cognitifs avérés et progressivement croissants. À ce stade, il est plus facile d'objectiver une inflexion de la pente de déclin. Cependant, la méta-analyse n'apporte guère d'éléments en faveur d'une supplémentation par I'EPA ou le DHA chez les sujets présentant des troubles cognitifs avérés mais pas déments (Mazereeuw et al., 2012). Au stade de la démence, toutes les études de supplémentation avec des oméga-3 seuls se sont avérées négatives (Cunnane et al., 2009 ; Quinn et al., 2010).

Le stade préclinique ou clinique sur lequel porte l'intervention conditionne aussi le choix du critère de jugement principal. L'incidence de la démence est trop faible chez des sujets sans symptômes cognitifs pour être utilisable dans des essais de durée nécessairement limitée. Un test global comme le MMSE est peu sensible au changement et se heurte à un effet plafond chez les sujets avec un bon niveau de fonctionnement cognitif. Les études d'intervention ont donc utilisé des batteries de tests neuropsychologiques très variables comme critère de jugement, avec parfois même des scores composites par domaine cognitif : mémoire, fonctions exécutives, fluence verbale... Cette hétérogénéité entre études limite la comparabilité des résultats et les possibilités de méta-analyse.

Des critères d'inclusion nutritionnels devraient aussi être pris en compte : il n'est sans doute pas utile de supplémenter en EPA et DHA des sujets ayant déjà des apports alimentaires suffisants. Dans l'ensemble des 8085 sujets âgés de l'étude $3 \mathrm{C}$ habitant à leur domicile à Bordeaux, Dijon ou Montpellier, $50 \%$ consommaient du poisson au moins deux fois par semaine et couvraient donc théoriquement leurs besoins en EPA et DHA (BarbergerGateau et al., 2007). Dans le souséchantillon bordelais incluant 1786 participants qui ont eu une enquête alimentaire par rappel des 24 heures, la consommation moyenne d'EPA plus DHA s'élevait à 420 mg/j (Feart et al., 2007), soit une quantité proche de la nouvelle recommandation française de $500 \mathrm{mg} / \mathrm{j}$ (Afssa, 2010) mais avec une très grande variabilité entre sujets. II serait souhaitable de $n^{\prime}$ inclure dans les études $d$ 'intervention que des participants ayant des apports insuffisants en EPA et DHA. Cependant l'étude de Quinn et al. (Quinn et al., 2010) qui a inclus des patients atteints de maladie d'Alzheimer légère à modérée qui consommaient moins de $200 \mathrm{mg} / \mathrm{j}$ de 
DHA en moyenne n'a pas mis en évidence de bénéfice d'une supplémentation avec $2 \mathrm{~g} / \mathrm{j}$ de DHA pendant 18 mois.

\section{Quelles quantités d'EPA et DHA apporter ?}

Les quantités optimales d'EPA et DHA et leur ratio à apporter dans un supplément pour prévenir ou retarder le déclin cognitif ne sont pas connus. Contrairement aux essais thérapeutiques avec des médicaments dont la molécule n'est pas présente dans l'organisme avant le traitement, les essais avec des suppléments nutritionnels sont réalisés chez des individus ayant un niveau basal variable en fonction de leur consommation alimentaire, et variant d'un jour à l'autre surtout pour des nutriments comme I'EPA ou le DHA provenant d'aliments comme le poisson dont la consommation n'est pas quotidienne. II existe donc une importante variabilité inter- et intra-individuelle dans l'exposition alimentaire aux oméga-3, à laquelle vient se surajouter la supplémentation. Cette variabilité tend à sous-estimer la force des associations avec la variable à expliquer, ici le déclin cognitif.

Des quantités très variables d'EPA et/ou de DHA ont été utilisées dans les études d'intervention. Parmi les six études analysées par Mazereeuw ayant porté chez des sujets avec des fonctions cognitives relativement préservées, les quantités d'EPA variaient de 0 à $1093 \mathrm{mg} / \mathrm{j}$ et celles de DHA de 59 à $1700 \mathrm{mg} / \mathrm{j}$, sans relation nette entre la dose et I'efficacité (tableau 1) (Mazereeuw et al., 2012). Ces études ne permettent pas non plus d'établir un ratio EPA/DHA optimal pour la prévention du déclin cognitif.

\section{Faut-il tenir compte des caractéristiques génétiques?}

Certaines études d'observation mais aussi d'intervention (Quinn et al., 2010) montrent des interactions avec I'ApoE4 : les sujets ne possédant pas cet allèle semblent mieux répondre à l'ingestion d'EPA ou DHA (Barberger Gateau et al., 2011, Plourde et al., 2009). Les mécanismes sous-jacents sont encore mal connus. Les porteurs de I'ApoE4 pourraient avoir une altération du transport des acides gras ou du cholestérol, du métabolisme des oméga-3, du glucose ou des cétones, ou d'autres modifications comme une exacerbation de I'inflammation cérébrale qui les rendraient plus résistants à l'action des oméga-3, à niveau de fonctionnement cognitif égal par ailleurs (Barberger Gateau et al., 2011). II est possible que d'autres polymorphismes génétiques qui jouent un rôle mineur dans le risque de maladie d'Alzheimer en population générale puissent aussi interagir avec les oméga-3. En effet, CLU (apolipoprotéine J), dont le SNP rs11136000 est associé à un risque diminué de maladie d'Alzheimer, est l'une des apolipoprotéi- nes les plus abondamment exprimées dans le système nerveux central, suggérant un rôle potentiel dans le transport ou la fonctionnalité des lipides.

\section{Serait-il plus efficace d'associer plusieurs nutriments?}

Enfin, un nutriment n'est jamais consommé de façon isolée dans l'alimentation. Il faut donc tenir compte des synergies et interactions avec les autres nutriments, en particulier les antioxydants. Ces derniers pourraient contribuer à protéger les oméga-3 à longue chaîne contre la peroxydation lipidique à laquelle ils sont très sensibles. Une forte adhérence au régime méditerranéen qui associe des consommations importantes de fruits, légumes, légumineuses, céréales, poisson et acides gras monoinsaturés provenant de I'huile d'olive a été associée à un moindre risque de déclin cognitif et de survenue de maladie d'Alzheimer dans plusieurs études épidémiologiques (Feart et al., 2010). Ce régime alimentaire apporte des vitamines anti-oxydantes $C$ et $E$, de la vitamine $B 6$ et des folates, qui pourraient avoir un rôle protecteur contre le déclin cognitif (Feart et al., 2012). Une forte adhérence au régime méditerranéen est également associée à des concentrations plasmatiques plus élevées en EPA et DHA (Barberger Gateau et al., 2011). II est donc difficile de distinguer le rôle spécifique d'un nutriment donné dans I'alimentation et on peut même faire

Tableau 1. Quantités d'EPA et de DHA utilisées dans les essais contrôlés randomisés réalisés chez des sujets à haut niveau de fonctionnement cognitif (MMSE > 25) inclus dans la méta-analyse de (Mazereeuw et al. (2012)).

\begin{tabular}{|c|c|c|c|c|}
\hline \multirow{2}{*}{$\begin{array}{l}\text { Étude : } \\
\text { Premier auteur (année) }\end{array}$} & \multicolumn{2}{|c|}{ Nombre de participants inclus } & \multicolumn{2}{|c|}{ Quantité administrée dans le supplément $(\mathrm{mg} / \mathrm{j})$} \\
\hline & $\begin{array}{l}\text { Groupe } \\
\text { intervention }\end{array}$ & $\begin{array}{l}\text { Groupe } \\
\text { placebo }\end{array}$ & EPA & DHA \\
\hline Dangour (2010) & 375 & 369 & 200 & 500 \\
\hline Freund-Levi (2006) & 16 & 16 & 600 & 1700 \\
\hline $\begin{array}{l}\text { Johnson } \\
\text { (2008) }\end{array}$ & 14 & 10 & 0 & 800 \\
\hline Vakhapova (2010) & 40 & 38 & 20 & $\begin{array}{l}59 \\
\text { (phosphatidyl sérine) }\end{array}$ \\
\hline Yurko-Mauro (2010) & 219 & 218 & 0 & 900 \\
\hline \multirow[t]{3}{*}{ Van de Rest (2008) } & 96 & 106 & 1093 & 847 \\
\hline & & & $\mathrm{Ou}$ & $\mathrm{Ou}$ \\
\hline & 100 & & 226 & 176 \\
\hline
\end{tabular}


I'hypothèse que les nutriments agissent en synergie.

Contrairement au domaine des maladies cardiovasculaires (De Lorgeril et al., 1994), il n'existe pas d'étude d'intervention ayant montré l'impact sur le déclin cognitif d'une modification du régime alimentaire pour se rapprocher d'une diète méditerranéenne. Cependant, la combinaison de nutriments destinés à améliorer le fonctionnement cognitif de sujets atteints de maladie d'Alzheimer légère ou modérée a été évaluée au sein du complément nutritionnel oral Souvenaid qui associe $300 \mathrm{mg}$ d'EPA et $1200 \mathrm{mg}$ de DHA mais aussi de la choline, des vitamines C, E, B6, B12, du sélénium et de l'acide folique, entre autres composants (Scheltens et al., 2010). L'essai contrôlé randomisé contre placebo Souvenir Il a évalué l'efficacité de Souvenaid chez des patients atteints de maladie d'Alzheimer modérée non traités (Scheltens et al., 2012). Au bout des 24 semaines de l'essai les 103 patients traités par Souvenaid avaient une amélioration significative du domaine de la mémoire de la batterie de tests neuropsychologiques, le critère de jugement principal, comparativement aux 103 sujets du groupe placebo isocalorique $(125 \mathrm{kcal} / \mathrm{j})$. Même si cet essai n'a pas montré d'impact sur les fonctions exécutives ou l'autonomie fonctionnelle, il constitue une preuve de concept de la possibilité d'une amélioration des fonctions cognitives avec une combinaison de nutriments chez des patients atteints de maladie d'Alzheimer.

Il faut également tenir compte du statut énergétique du cerveau. En effet, certaines régions du cerveau vieillissant ne seraient pas aussi efficaces pour extraire le glucose de la circulation que chez un adulte jeune. Les individus à risque génétique de maladie d'Alzheimer ont une diminution de la captation du glucose avant les premiers signes de déclin cognitif (Cunnane et al., 2011). Lorsque les troubles cognitifs sont avérés, il semble que la captation du glucose soit définitivement compromise, en particulier dans le cortex temporal (Mamelak, 2012). Dans ces conditions, le statut énergétique des neurones ou des circuits cérébraux impliqués dans le déficit cognitif serait un facteur limitant l'efficacité les suppléments d'EPA ou DHA. L'insulino-résistance et le diabète sont d'importants facteurs de risque de déclin cognitif chez le sujet âgé et semblent aussi contribuer à détériorer la captation du glucose par le cerveau (Baker et al., 2011). II serait donc nécessaire de concevoir et évaluer des interventions permettant d'optimiser ou de restaurer le métabolisme énergétique cérébral en même temps que d'apporter des oméga-3 ou d'autres nutriments essentiels pour la prévention du déclin cognitif du sujet âgé.

\section{Conclusion}

Les études épidémiologiques d'observation et les études d'intervention ont donné des résultats discordants quant à l'intérêt des oméga-3 à longue chaîne dans la prévention ou le traitement du déclin cognitif du sujet âgé. En l'absence d'essai contrôlé randomisé probant, il existe encore de nombreuses incertitudes sur l'impact réel d'une supplémentation en EPA ou DHA. La recherche doit donc se poursuivre pour mieux identifier les bénéficiaires potentiels d'une supplémentation, en fonction de leurs caractéristiques cognitives, nutritionnelles, voire génétiques. Les quantités et proportions optimales d'EPA et de DHA, ainsi que $d^{\prime}$ autres nutriments pouvant potentialiser leur effet, doivent être déterminées. Les interactions entre nutriments et gènes impliqués dans le risque de maladie d'Alzheimer doivent être explorées. Ces étapes sont un préalable indispensable avant la mise en place de nouvelles études d'intervention bien conduites ciblant les individus les plus à risque de déclin cognitif.

Conflits d'intérêts : PBG : participation en tant que co-investigateur à l'étude MAPT (Multidomain Alzeimer Prevention Trial). Interventions ponctuelles: Lesieur, Danone, Bausch \& Lomb, Aprifel, Groupe Lipides et Nutrition. Versements substantiels au budget d'une institution dont elle est responsable: Danone, THEA, Vifor pharma, Groupe Lipides et Nutrition. CF : interventions ponctuelles: Conférence IOFECCEO 2012 - invitée par Danone Research. CS et SC : aucun.

\section{RÉFÉRENCES}

Amieva H, Le Goff $M$, Millet X, et al. Prodromal Alzheimer's disease: Successive emergence of the clinical symptoms. Ann Neurol 2008 ; 64 : 492-8.
Baker LD, Cross DJ, Minoshima S, Belongia D, Watson GS, Craft S. Insulin resistance and Alzheimer-like reductions in regional cerebral glucose metabolism for cognitively normal adults with prediabetes or early Type 2 diabetes. Arch Neurol 2011 ; 68 : 51-7.

Barberger-Gateau P, Raffaitin C, Letenneur L, et al. Dietary patterns and risk of dementia: the Three-City cohort study. Neurology 2007 ; $69: 1921-30$.

Barberger-Gateau P, Samieri C, Allès B, Féart C. Could nutrition prevent the onset of dementia? Current evidence from epidemiological and intervention studies. Neurodegener Dis Manag 2012 ; 2 : 305-14.

Barberger Gateau P. PUFAs and risk of cognitive decline or dementia: epidemiological data. OCL 2007 ; 14 : 198-201.

Barberger Gateau P, Feart C, Samieri C. Mediterranean diet and cognitive decline: what role for omega-3 polyunsaturated fatty acids? OCL $2011 ; 18$ : 224-7.

Barberger Gateau P, Samieri C, Feart C, Plourde M. Dietary omega 3 polyunsaturated fatty acids and Alzheimer's disease: Interaction with apolipoprotein E genotype. Curr Alzheimer Res 2011 ; 8 : 479-91.

Cunnane S, Nugent S, Roy M, et al. Brain fuel metabolism, aging, and Alzheimer's disease. Nutrition $2011 ; 27$ : 3-20.

Cunnane SC, Plourde M, Pifferi F, Bégin $M$, Féart C, Barberger-Gateau P. Fish, docosahexaenoic acid and Alzheimer's disease. Prog Lipid Res 2009 ; 48 : 239-56.

Dangour AD, Andreeva VA, Sydenham E, Uauy R. Omega 3 fatty acids and cognitive health in older people. Br J Nutr 2012; 107 (Suppl. 2) : S152-8.

De Lorgeril M, Renaud S, Mamelle N, et al. Mediterranean alpha-linolenic acid-rich diet in secondary prevention of coronary heart disease. Lancet 1994 ; 343 : 1454-9.

Feart C, Alles B, Merle B, Samieri C, Barberger-Gateau P. Adherence to a Mediterranean diet and energy, macro-, and micronutrient intakes in older persons. I Physiol Biochem $2012 ; 68$ : 691-700.

Feart C, Jutand MA, Larrieu S, et al. Energy, macronutrient and fatty acid intake of French elderly community dwellers and association with socio-demographic characteristics: data from the Bordeaux sample of the Three-City Study. Br / Nutr 2007 ; 98 : 1046-57.

Feart C, Samieri C, Barberger-Gateau P. Mediterranean diet and cognitive function in older adults. Curr Opin Clin Nutr Metab Care $2010 ; 13$ : 14-8.

Jack JR CR, Knopman DS, Jagust WJ, et al. Hypothetical model of dynamic biomarkers 
of the Alzheimer's pathological cascade. Lancet Neurol $2010 ; 9$ : 119-28.

Mamelak M. Sporadic Alzheimer's disease: the starving brain. J Alzheimers Dis 2012; 31 : 459-74.

Mazereeuw G, Lanctot KL, Chau SA, Swardfager W, Herrmann N. Effects of omega-3 fatty acids on cognitive performance: a meta-analysis. Neurobiol Aging 2012 ; 33 : 1482.e17-29.

Plourde M, Vohl M-C, Vandal M, Couture P, Lemieux S, Cunnane SC. Plasma n-3 fatty acid response to an n-3 fatty acid supplement is modulated by apoE 4 but not by the common PPAR-alpha L162V polymorphism in men. Br J Nutr 2009 ; 102 : 1121-4.

Profenno LA, Porsteinsson AP, Faraone SV. Meta-analysis of Alzheimer's disease risk with obesity, diabetes, and related disorders. Biol Psychiatry $2010 ; 67$ : 505-12.
Quinn JF, Raman R, Thomas RG, et al. Docosahexaenoic acid supplementation and cognitive decline in Alzheimer disease: A randomized trial. JAMA 2010 ; 304 : 190311.

Ramaroson H, Helmer C, Barberger-Gateau $P$, Letenneur $L$, Dartigues JF. Prévalence de la démence et de la maladie d'Alzheimer chez les personnes de 75 ans et plus : données réactualisées de la cohorte PAQUID. Rev Neurol 2003 ; 159 : 405-11.

Ritchie K, Artero S, Touchon J. Classification criteria for mild cognitive impairment: a population-based validation study. Neurology $2001 ; 56: 37-42$.

Samieri C, Maillard P, Crivello F, et al. Plasma long-chain omega- 3 fatty acids and atrophy of the medial temporal lobe. Neurology $2012 ; 79: 642-50$.
Scheltens P, Kamphuis PJGH, Verhey FRJ, et al. Efficacy of a medical food in mild Alzheimer's disease: A randomized, controlled trial. Alzheimers Dement 2010 ; 6 : 1-10.e1.

Scheltens P, Twisk JW, Blesa R, et al. Efficacy of souvenaid in mild Alzheimer's disease: results from a randomized, controlled trial. J Alzheimers Dis 2012 ; 31 : 225-36.

Sydenham E, Dangour AD, Lim WS. Omega 3 fatty acid for the prevention of cognitive decline and dementia. Cochrane Database Syst Rev $2012 ; 6$ : CD005379.

Viswanathan A, Rocca WA, Tzourio C. Vascular risk factors and dementia: How to move forward? Neurology $2009 ; 72$ : 368-74.

Yurko-Mauro K, Mccarthy D, Rom D, et al. Beneficial effects of docosahexaenoic acid on cognition in age-related cognitive decline. Alzheimers Dement 2010 ; 6 : 456-64. 